\title{
Evidence of zonular stress lines on human crystalline
} lens

\begin{abstract}
Crystalline lens and zonules play an important role in accommodation. There are various clinical and theoretical explanations of tension zonules and its role in accommodative changes of the lens. We present the morphology of zonular stress lines (ZSLs) under bright field compound microscopy on the surfaces of transparent crystalline lenses in three human enucleated eyeballs removed for varied indications. ZSLs were evident on the periphery and paracentral part of crystalline lenses. Further study on these stress lines with advance imaging will give more important clue in the complex zonule-lens morphology.
\end{abstract}

Keywords: zonules, lens, enucleation, accomodation
Volume 8 Issue 3 - 2018

\author{
Dipankar Das, Harsha Bhattacharjee, Diva \\ kant Misra, Panna Deka, Fazil Khurrum, \\ Saurabh Deshmukh, Puneet Mishra, Priyank \\ Bhola, Apurba Deka \\ Sri Sankaradeva Nethralaya, India
} Pathology, Uveitis \& Neuro-Ophthalmology Services, Sri Sankaradeva Nethralaya, Beltola, Guwahati-781028, Assam, India, Tel +036I-2228879, 23055 I6, Fax \#036I- 2228878, Emaildr_dasdipankar@yahoo.com

Received: May 31, 2018 | Published: June 15, 2018

\section{Introduction}

The lens is an extremely planned system of focused cells which constitutes an important element of optical arrangement of the eye and fulfills the important functions of altering the refractive index of light entering the eye to focus on the retina. ${ }^{1,2}$ The transparency of lens is due to the shape, array, internal structure, and biochemistry of the lens cells or lens fibre. ${ }^{1}$ The lens is held in place by composite three dimensional systems of radially arranged zonules called zonules of Zinn or the suspensory ligament of the lens. ${ }^{1}$ These fragile fibres are attached to the lens capsule $2 \mathrm{~mm}$ anterior and $1 \mathrm{~mm}$ posterior to the equator and arise from the region of pars plana ciliary epithelium and pass forward closely related to the lateral surfaces of ciliary processes. ${ }^{1}$ The fibrous zonules blend with basal lamina of the lens capsule. The anterior and posterior zonules place in obliquely into superficial $1-2 \mu \mathrm{m}$ of preand post-equatorial lens capsule, while equatorial zonules insert at right angles. ${ }^{1-3}$ We present the documentation of zonular stress lines (ZSLs) for the first time in the scientific literature on the transparent crystalline lens from three enucleated eyeballs. These stress lines are the evidence of the zonules to take part in accommodation and also arbitrate accommodative movements. Although some believed that there are two types of zonules, first being 'main zonules' and other the 'tension zonules', the later being placed under tension during accommodation but there were no morphological different stress lines on the surface of lens in so called two varieties of zonules. ${ }^{1-3}$

\section{Case series}

This was a retrospective, observational and laboratory based study. Three crystalline lenses from three enucleated eyes for varied other indications were examined in ocular pathology laboratory in a tertiary institute of Northeast India. The informed consents were taken before the surgeries.

\section{Case I}

A 4 month old female child, clinically diagnosed as Group E retinoblastoma of the right eye. After enucleating the eye, gross examination of the eyeball was carried out and portion of transparent crystalline lens was brought out for direct examination under the bright-field compound microscope (Zeiss, AxioCam, MRc in Axioskop 40). An overview of stress lines of zonules on the surface of lens showed interesting observation. ZSLs were observed in the paracentral portion and at the periphery of the lens. At the periphery, there were angulations of the stress lines which were documented (Figure $1)$.

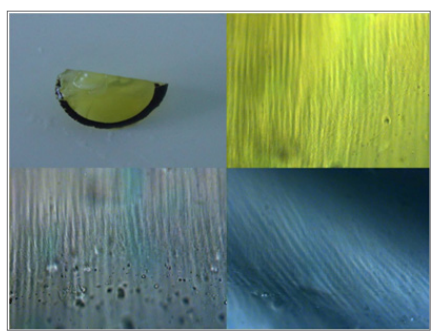

Figure I Specimen I with gross photo of transparent crystalline lens.Adjacent microscopic picture showed the ZSLs visualized directly under compound microscope (Zeiss, AxioCam, MRc in Axioskop 40). Bending of the stress lines obliquely could also be seen in the inset $(x 400)$.

\section{Case 2}

A 4 year old male child presented with white pupillary reflex of the right eye. A provisional diagnosis of retinoblastoma or Coat's disease was made. Gross and microscopic examination of the specimen was consistent with Coat's disease of the eyeball. Surface of the lens showed stress lines and were subsequently recognized (Figure 2)

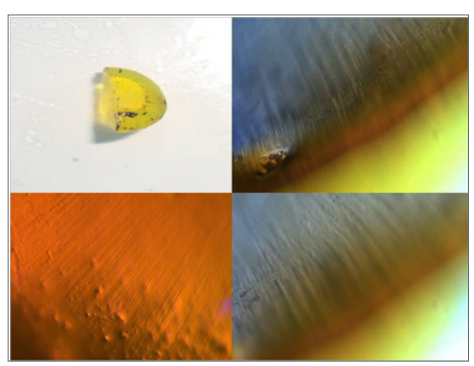

Figure 2 Gross lens photo and microscopic evidence of ZSLs in specimen 2 (x 400). 


\section{Case 3}

A 32 years old male patient presented to the institute with a history of penetrating trauma of the left eye and subsequently developed painful blind eye. Enucleation of that eye was done and after overnight fixation in $10 \%$ neutral buffered formalin, the eyeball was sectioned vertically. Lens was seen separately and ZSLs were observed on the anterior surface of the lens and brownish iris pigmentation was seen on the same surface with orientation of pigmentation along the stress lines (Figure 3). Microscopic examination of the eyeball showed there was evidence of endophthalmitis and special stains were carried out to know the causative organisms. We could clearly identify the presence of clefts by zonules on the surface of lenses particularly at the periphery of lenses with its extension to the para-central areas. They were multilayered and created linear impression on the surfaces of the lenses (Figures 1-4).

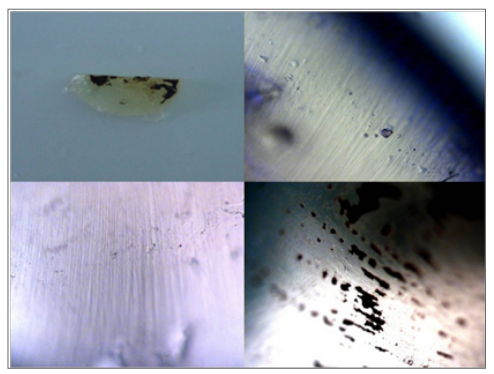

Figure 3 Specimen 3 with gross and microscopic impression of ZSLs. Note the pigmentation on the anterior surface of lens was oriented along the stress lines $(x 400)$.

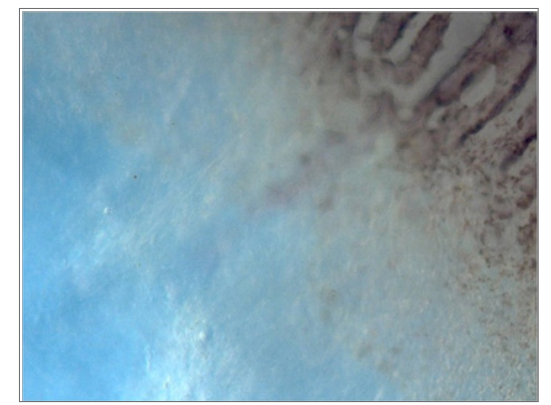

Figure 4 Zonular insertion of crystalline lens without stress lines on it, I00X, Ol.

\section{Discussion}

The lens of the eye is a clear, biconvex, oval, semisolid, avascular body of crystalline structure located between the iris and the vitreous. ${ }^{4}$ The lens is unique among organs in that it contains cells exclusively of a single type, in various stages of cyto-differentiation and retains within it all the cells formed during its life time. ${ }^{4,5}$ Ciliary zonule consists basically of a series of fibers passing from the ciliary body to the lens. ${ }^{4,5}$ It holds the lens in place and helps ciliary muscle to act on it during accommodation. ${ }^{4}$ The zonules consist of thick, smooth bundles 5-30 $\mu \mathrm{m}$ in diameter. Each bunch consists of a series of fine fibres $\left(0.35-1 \mu \mathrm{m}\right.$ in diameter) and they composed of $8-12 \mu \mathrm{m}$ fibrils. ${ }^{1-4}$ One potentially applicable consequence of continued lens growth relates to altered zonulo-lenticular-ciliary body geometry and the resulting changes in degree and vector of the ciliary body and choroidal forces applied on the lens. ${ }^{5-8}$ Since the axial posterior surface of the lens remains permanent in position relative to the cornea and retina while lens grows, the central sulcus bisecting the lens equatorially is translated anteriorly, as is the actual lens equator. ${ }^{5-8} \mathrm{We}$ had established the evidence of ZSLs on the anterior surface of the transparent crystalline lens in three specimens. These stress lines have clefts in a characteristic pattern on the surface of lenses which were prominent at the periphery and they could be traced towards the para-central areas. One can hypothesize that many optical consequences to these changes and even the anterior shift of the lenses could increase these ZSLs or zonular tension on the anterior capsule, making accommodation more difficult. ${ }^{9,10} \mathrm{We}$ had seen that younger lenses in our series had less prominent ZSLs while lens seen in 32 year old man had more prominent ZSLs marking on its surface.

\section{Acknowledgements}

Sri Kanchi Sankara Health and Educational Foundation.

\section{Conflict of interest}

The Author declares that there is no conflict of interest.

\section{References}

1. Forrester JV, Dick AD, McMenamin P. The Eye: Basic Sciences in Practice. $2^{\text {nd }}$ ed. Philadelphia: WB Saunders; 2001.

2. Brown N. The change in shape and internal form of the lens of the eye on accommodation. Exp Eye Res. 1973;15(4):441-459.

3. Pedrigi RM, David G, Dziezyc J, et al. Regional mechanical properties and stress analysis of the human anterior lens capsule. Vision Res. 2007;47(13):1781-1789

4. Bron A, Tripathi R, Tripathi B. Wolff's anatomy of the eye and orbit. $8^{\text {th }}$ ed. London: Chapman and Hall; 1997.

5. Manns F, Prel JM, Denham D, et al. Optomechanical response of human and monkey lenses in a lens stretcher. Invest Ophthalmol Vis Sci. 2007;48(7):3260-3268.

6. Drecoll EL, Kaufman PL, Wasielewski R, et al. Morphology and accommodative function of the vitreous zonule in human and monkey eyes. Invest Ophthalmol Vis Sci. 2010;51(3):1554-1564.

7. Hart WM. Adler's Physiology of the Eye: Clinical Application. $9^{\text {th }}$ ed. St. Louis: Mosby; 1992.

8. Koretz JF, Bertasso AM, Neider MW, et al. Slit-lamp studies of the rhesus monkey eye: II. Changes in crystalline lens shape, thickness and position during accommodation and aging. Exp Eye Res. 1987;45(2):317-320.

9. Heys KR, Cram SL, Truscott RJW. Massive increase in the stiffness of the human lens with age: the basis of presbyopia? Mol Vis. 2004;10:956-963.

10. Weeber HA, Eckert G, Pechhold W, et al. Stiffness gradient in the crystalline lens. Graefes Arch Clin Exp Ophthalmol. 2007;245(9):13571366. 